\title{
Mechanisms for surface potential decay on fluorinated epoxy in high voltage DC applications
}

\author{
A. Mohamad*¹, G. Chen ${ }^{1}$, Y. Zhang ${ }^{2}$ and Z. $\mathrm{An}^{2}$ \\ ${ }^{1}$ School of Electronics and Computer Science, University of Southampton, SO17 1BJ, United Kingdom \\ ${ }^{2}$ Department of Electrical Engineering, Tongji University, Shanghai, China \\ *E-mail: am306r@ecs.soton.ac.uk
}

\begin{abstract}
Epoxy resin has been extensively used for decades as an insulation material in high voltage transmission systems. However, this insulation material does suffer from bulk and surface charging when used as insulating spacer, mainly in high voltage DC applications. By applying fluorination treatment, the surface of polymeric insulation is chemically treated and so modifies charge transport characteristics of the material. In doing so, excellent surface properties can be obtained without compromising the bulk characteristics of the polymeric insulation. In this paper, the authors investigate the surface potential decay performance of non-fluorinated and fluorinated epoxy resin samples. The surface decay performance of insulating material is a crucial parameter in dissipating accumulation of surface and bulk charge that can lead to premature breakdown of the insulating material. The epoxy samples were characterised by Energy Dispersive X-Ray (EDX) analysis to determine the changes in chemical composition of the samples before and after fluorination treatment. Surface potential decay measurement using positive corona discharging was then performed, followed by bulk DC conductivity measurement to further explain the mechanisms which govern the surface potential decay. The existence of surface-fluorinated layer on the treated samples had been found to play a major role in dictating the movement of charges away from the surface during the decay process. The influence of fluorination treatment on the decay mechanisms was discussed.
\end{abstract}

\section{INTRODUCTION}

Recent studies on insulating material treatment tend to focus on the modifications of bulk insulating material [1-3], which lead to changes in the properties of the bulk, as well as the surface layer, and consequently its interface properties with electrode. However, little attention has been given to modifications limited to surface properties alone by directly treating the surface layer of polymeric insulation. The idea of this paper is to perform chemical treatment on the surface of polymeric insulation and so modify charge transport characteristics of the subjected material via fluorination treatment. This chemical treatment process has been well developed from fundamental concepts to industrial applications and is amongst the most effective approaches to improving materials in term of barrier properties, separation properties, thermal and chemical stability, as well as biocompatibility [4-6].

Through fluorination treatment, excellent surface properties comparable to those of fluoropolymers can be achieved without compromising the bulk characteristics of the virgin insulating material. The usages of fluorination treatment so far are mostly concentrated on improving properties with regards to adhesion, wettability, biocompatibility, barrier properties, chemical stability, and grafting [7]. There has been far too little attention given to modification of dielectric properties. In fact, the modifications in the chemical components of polymeric insulation should also lead to corresponding modifications in dielectric properties of the insulating material [8].

In this paper, epoxy resin samples were formulated and undergone fluorination treatment. The samples were then characterised by Energy Dispersive X-Ray (EDX) analysis to determine its chemical composition. Finally, the surface potential decay and the DC conductivity test were carried out in order to gauge the surface decay performance of the treated insulating material as well as to further explain the mechanisms behind the surface potential decay which took place.

\section{EXPERIMENTAL DETAILS}

\section{A. Materials and treatment}

A set of Bisphenol-A type epoxy resin (Araldite LY556) with anhydride hardener (Aradur 917) and imidazole accelerator (DY070) from Huntsman Advanced Materials (with weight ratio $100: 90: 2$ ) were used throughout this study. These components were degassed separately inside vacuum oven $\left(10^{3} \mathrm{~Pa}\right.$ at $\left.40^{\circ} \mathrm{C}\right)$ for 30 minutes and another 10 minutes after mixing. The mixture was filled into a stainless steel mould, which had been applied with release agent QZ13 to help release the cured sample from the mould when the curing is done. This release agent enables precise reproduction of surface detail and does not corrode araldite epoxy or metal moulds. A melamine film of $250 \mu \mathrm{m}$ thickness was used as a spacer in between the moulds in order to produce $250 \mu \mathrm{m}$ thick epoxy sample. The mould was then placed in the oven at $80^{\circ} \mathrm{C}$ for 4 hours for gelation process. The mould was again heated at $120^{\circ} \mathrm{C}$ for another 8 hours for post-cured process. Once the epoxy was fully cured and taken out from the mould, it was cut into $40 \mathrm{~mm} \times 40 \mathrm{~mm}$ size for experimental purposes. This epoxy system produces a high-molecular-weight polymer that can combine thermoplastic processability with a robust mechanical behaviour, adhesion to a variety of active surfaces, attractive optical characteristics and an exceptional barrier to oxygen and other atmospheric gases [9].

The above produced samples were then fluorinated with varying time duration. Fluorination of the samples was done in a closed stainless vessel. After evacuation, the fluorine and nitrogen mixture of ratio 1:4 flowed into the vessel. The gas 
pressure in the vessel is kept at 225 mbars at $50^{\circ} \mathrm{C}$. Two different fluorination times were performed; 30 minutes and 60 minutes. After reaction, the reactive gases were purged from the vessel with nitrogen.

\section{B. Energy Dispersive X-Ray Analysis}

EDX utilizes the X-ray spectrum produced by a subject bombarded with a focused beam of electrons to achieve a localized chemical spectrum. In theory, all elements from atomic number 4 (Be) to 92 (U) can be spotted [10]. Qualitative analysis, i.e. the determination of the peaks in the spectrum is fairly straightforward due to the minimalism of Xray spectra. Quantitative analysis, i.e. the determination of the concentrations of the elements present involves assessing peak strengths for each element in the sample.

The prepared samples were cut into small pieces of $10 \mathrm{~mm} \mathrm{x}$ $10 \mathrm{~mm}$ and were gold coated before being put under the microscope. Figure 1 to Figure 3 shows the EDX spectrum of non-fluorinated, 30-minute-fluorinated and 60-minutefluorinated samples. Traces of gold $(\mathrm{Au})$ can be seen from the spectrums due to the gold coating of the samples. In Figure 1, peaks of carbon (C) and oxygen $(\mathrm{O})$ can be seen from the spectrum, which are the main elements for epoxy. However, another main element for epoxy, hydrogen, is not detectable due to the limitation of EDX in detecting elements of small atomic number. In Figure 2, peak for fluorin (F) can be seen from the spectrum because of the existence of the fluorinated layer from the fluorination treatment. The fluorin peak is greater in intensity in Figure 3, indicating a higher concentration of fluorin element in the 60-minute-fluorinated epoxy. Level of fluorination and thickness of the fluorinated layer depend upon the treatment parameters of the composition and pressure of the reactive mixture, fluorine partial pressure and treatment time, as well as temperature and nature of the polymer in use [11]. A Raman spectrum was conducted in author's previous paper to prove that a chemical reaction did take place on the surface of epoxy [12].

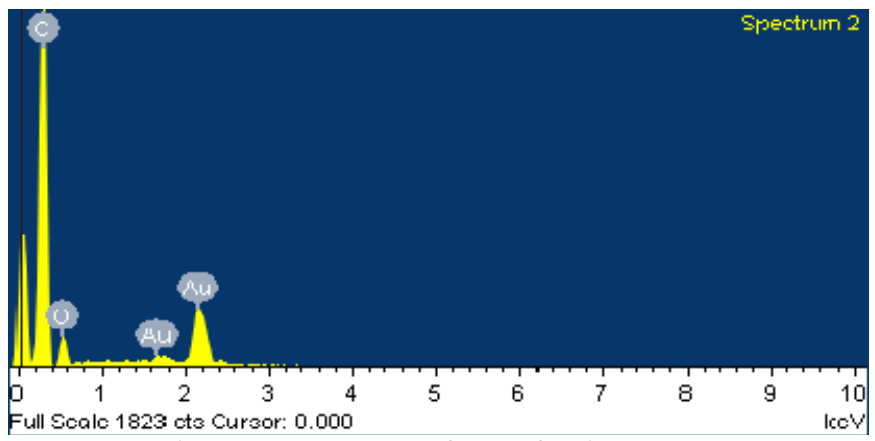

Figure 1: EDX spectrum for non-fluorinated epoxy

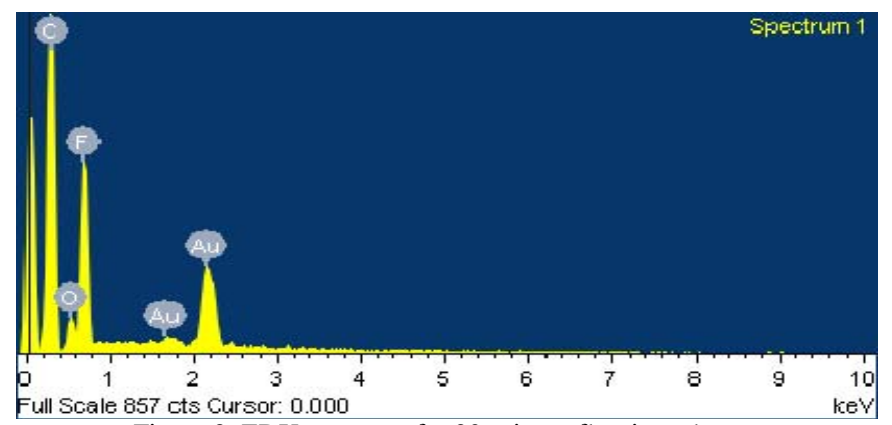

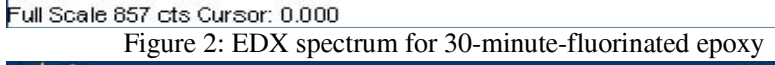

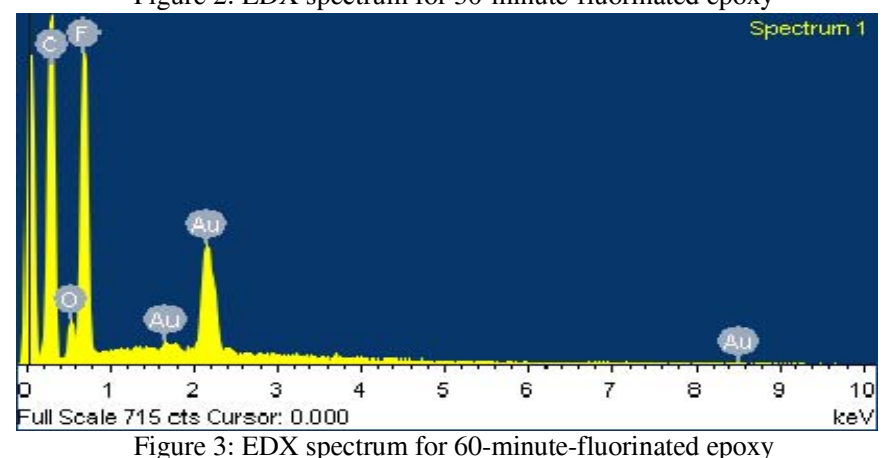

\section{Surface potential decay test}

Surface potential decay measurement enables a simple quantification of charge carrier mobility and trap parameters for each fluorination condition. This method involves charging the surface of an insulating material to a certain voltage and measuring the surface decay using a static monitor. The schematic diagram for the surface potential decay kit used is shown in Figure 4.

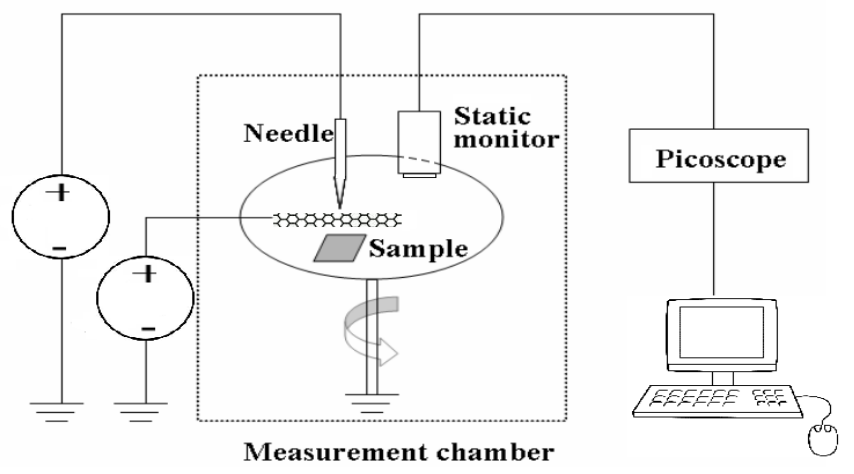

Figure 4: Surface potential decay schematic diagram

The epoxy samples were placed on top of a swivel-ground plate, just underneath a high voltage needle electrode and a wire-mesh-grid electrode. The distance between the needle and the grid is $4.5 \mathrm{~cm}$ while the distance between the grid and the ground plate is $1.5 \mathrm{~cm}$. The grid has a surface area of 150 $\mathrm{cm}^{2}$, sufficient to provide uniform distribution of corona charge on the sample surface of $16 \mathrm{~cm}^{2}(4 \mathrm{~cm} \mathrm{x} 4 \mathrm{~cm})$. The corona charging was done using a needle connected to a positive power supply, for a period of 1 minute, which deposited positive charges onto the surface of the material. The needle and grid voltage are $16 \mathrm{kV}$ and $5 \mathrm{kV}$ respectively. After charging, the sample was quickly moved towards a compact JCI 140 static monitor for surface potential 
measurement for 40 minutes duration. The surface potential decay against time characteristics was plotted.

The information from the result of this test, however, is insufficient to explain the mechanisms that govern the surface charge decay. DC conductivity measurement in the bulk may provide supporting evidence for the mechanisms responsible for the decay process.

\section{DC conductivity measurement}

The surface potential decay of epoxy samples seems to suggest that the surface conduction plays a leading role in the charge decay process. To further examine this argument, the DC conductivity measurement was used to observe the absorption current inside non-fluorinated and fluorinated epoxy samples. The schematic diagram of DC conductivity measurement is shown in Figure 5.

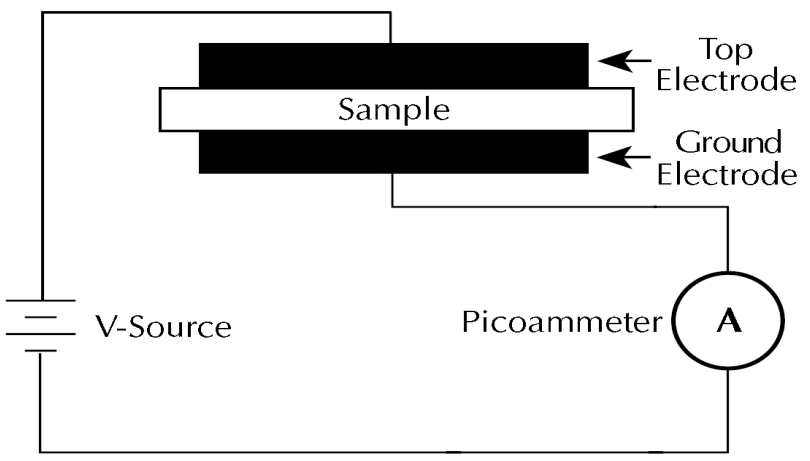

Figure 5: Schematic diagram of DC conductivity measurement

The top and ground electrodes are made of copper and are $30 \mathrm{~mm}$ in diameter, sandwiching the epoxy sample of $250 \mu \mathrm{m}$ in thickness. The electrodes were uniformly attached to the sample by a spring loaded to the bottom electrode. A constant DC voltage of $5 \mathrm{kV}$ was applied for a duration of 50 minutes at room temperature. As this analysis is used for comparison purposes only, the role of a grounding-ring electrode is neglected. The effects of leakage surface current for all measurements are deemed the same.

\section{RESULTS AND DISCUSSIONS}

From the surface potential decay measurement, different rates of surface potential decay can clearly be observed, as shown in Figure 6. The initial surface potential for all three samples is $\sim 2.8 \mathrm{kV}$. For non-fluorinated epoxy resin sample (F00), the surface potential decay is stable, indicating a very small amount of charges dissipates away from the surface. At the end of the 40 minutes decay time, the surface potential of F00 is $2.3 \mathrm{kV}$, a reduction of mere $0.5 \mathrm{kV}$ from initial charging. For the 30-minute-fluorinated sample $(\mathrm{F} 30)$, the decay rate is faster as the surface potential drops to $0.8 \mathrm{kV}$ after 40 minutes. The fastest decay rate can be seen for the 60-minutefluorinated sample (F60) signifying faster movement of charges away from the surface as the fluorination time increases. A significant drop of $2.7 \mathrm{kV}$ to $0.1 \mathrm{kV}$ can clearly be seen at the end of the measurement for F60. The observed phenomena occur because the fluorination treatment slightly improves the surface conductivity of epoxy samples [13]. The increase in conductivity is even more significant with prolonged time of fluorination treatment and, therefore, allows any surface charges to decay away faster.

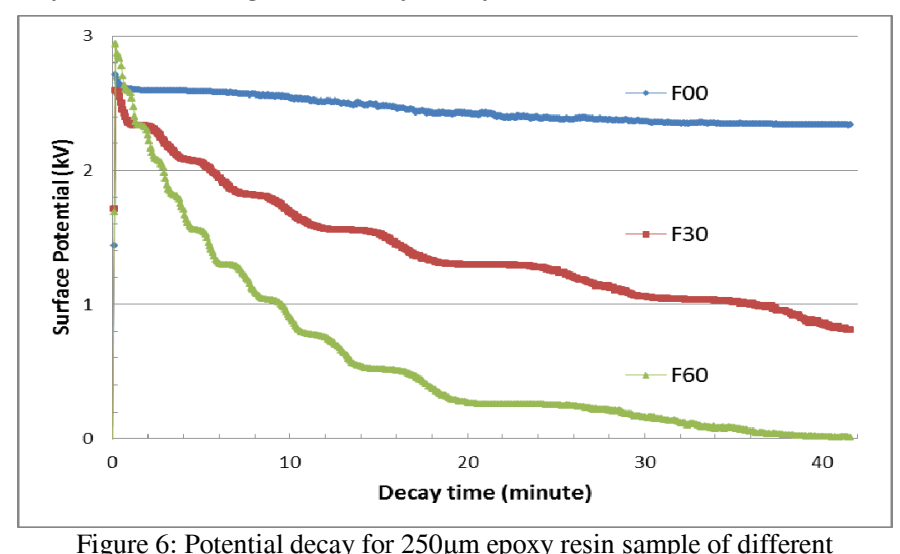

Figure 6: Potential decay for $250 \mu \mathrm{m}$ epoxy resin sample of different fluorination time

Studies show that there are three mechanisms that govern surface charge decay, which are (1) bulk conduction, (2) conduction along the surface and (3) neutralization by ion present in the gas volume above the sample surface [14]. The neutralization by ion mechanism may deem insignificant as all tests are conducted at atmospheric pressure in a controlled environment, leaving the possibility of surface charge decay to be through bulk conduction or diffusion along the surface.

Figure 7 shows plots of time dependence of leakage current for non-fluorinated sample (F00), 30-minute-fluorinated sample (F30), and 60-minute-fluorinated sample (F60). It is noted that, for all the samples, the value of the current drops significantly in the first few minutes of the measurement, due to the polarisation process taking effect which is a slow process in bulk and only plays a small contribution on the surface [15]. The dielectric relaxation current is also much higher in fluorinated samples due to the incorporation of polar groups in the form of carbon-fluorine bond on the surface layer.

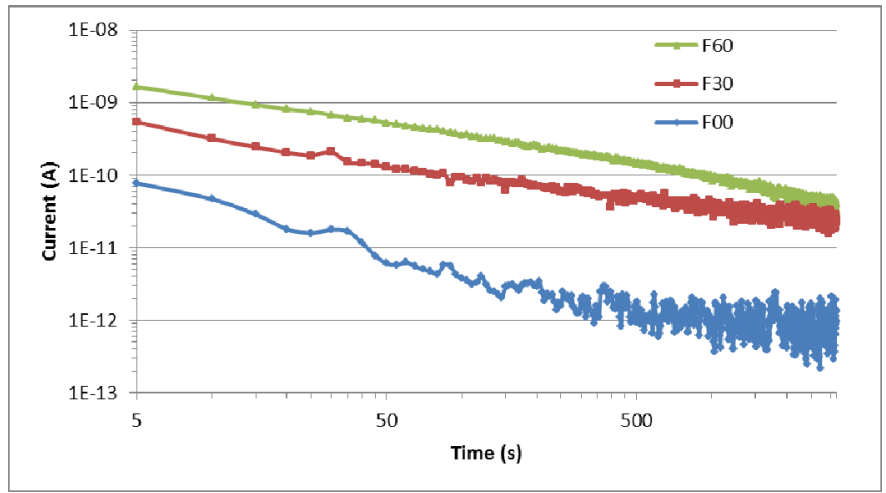

Figure 7: DC conductivity measurement for $250 \mu \mathrm{m}$ epoxy resin sample of different fluorination time

In non-fluorinated epoxy sample as illustrated in Figure 8, the absorption current that flows through the bulk of the sample is minimal and after 50 minutes, the bulk current is around $1.19 \mathrm{e}^{-12} \mathrm{~A}$. Where else, in the fluorinated epoxy sample 
as illustrated in Figure 9, there exists a new pathway for the current to flow in the form of fluorinated layer which possesses slightly higher conductivity than the bulk. The fluorinated layer covered the whole surface of the epoxy sample with thickness of $\sim 0.3 \mu \mathrm{m}$ for 30 -minute-fluorinated sample and $\sim 0.6 \mu \mathrm{m}$ for 60 -minute-fluorinated sample [12]. The leakage current significantly increases to $2.40 \mathrm{e}^{-11} \mathrm{~A}$ and $4.12 \mathrm{e}^{-11} \mathrm{~A}$ respectively after 50 minutes. In other words, with the introduction of surface-fluorinated layer, a big chunk of the current flows through this channel instead of the bulk with a current ratio of 20:1 for 30-minute-fluorinated sample and 35:1 for 60-minute-fluorinated sample.

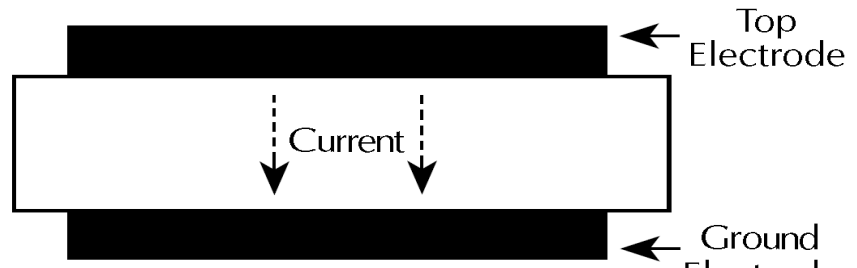

Figure 8: Minimal current flow through the bulk of non-fluorinated epoxy sample

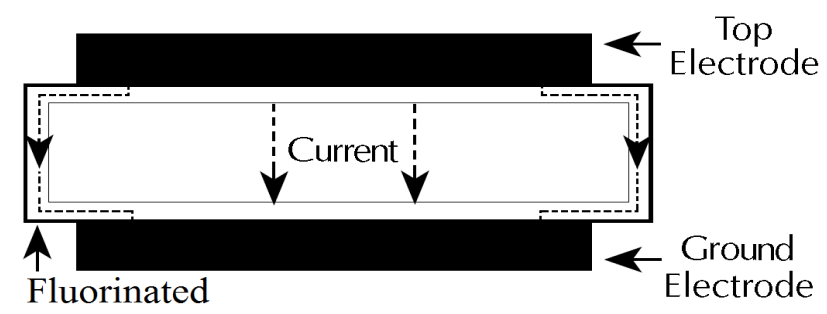

Layer

Figure 9: Extra current flow through the fluorinated layer in fluorinated epoxy sample

In relation to the surface potential decay measurement, the author suggests that the main mechanism that governs the surface potential decay is the conduction along the fluorinated surface layer, as seen from the current flow in the DC conductivity measurement. Only a small fraction of the current decays through bulk conduction. This is consistent with our earlier work showing that the fluorination treatment suppresses the charge injection from the bulk [13].

\section{CONCLUSIONS}

This paper looked into the effect of the incorporation of surface-fluorinated layer on epoxy resin sample in enhancing the surface potential decay characteristic of the insulating material. A commercial set of Bisphenol-A type epoxy resin was used throughout this study. The samples underwent fluorination treatment of varying time duration; 30 minutes and 60 minutes.

The epoxy samples were characterised using energy dispersive X-ray analysis in order to qualitatively and quantitatively determine the chemical composition of the virgin as well as the treated samples. It has been proven that the treated samples do contain fluorin element visible from the EDX spectrum in which the fluorin peaks intensifies with the treatment time.
The surface potential decay measurement of positive corona discharge verifies that the treated samples have better surface decay properties as compared to the virgin sample. From the DC conductivity test, it is proven that the trapped charges in surface decay mechanism diffuse along the surface of the fluorinated sample, with only a fraction of the charges flowing through the bulk. The fluorination treatment introduces a new surface layer which possesses slightly higher conductivity as compared to original epoxy, which offer a less resistive path for the charges to decay away from the surface. The incorporation of surface-fluorinated layer on epoxy resin appears to be a decisive factor in improving the dielectric properties of epoxy resin in high voltage DC applications.

\section{REFERENCES}

[1] Heung-Jin Ju, Kwang-Cheol Ko, Seung-Kil Choi "Optimal Design of a Permittivity Graded Spacer Configuration in a Gas Insulated Switchgear" Journal of the Korean Physical Society, vol.55, no 5, pp. 1803-1807, November

[2] Muneaki Kurimoto, Katsumi Kato, Masahiro Hanai, Yoshikazu Hoshina, Masafumi Takei, Hitoshi Okubo "Application of Functionally Graded Material for Reducing Electric Field on Electrode and Spacer Interface" IEEE Transactions on Dielectrics and Electrical Insulation, vol. 17, issue 1, pp.256-263, February

[3] Shengtao Li, Tuo Zhang, Qifeng Huang, Weiwei Li, Fengyan Ni, Jianying Li "Improvement of Surface Flashover Performance of Al2O3 Ceramics in Vacuum by Adopting A-B-A Insulation System" Plasma Science and Technology, vol. 13, no. 2, April 2011

[4] A.P. Kharitonov "Direct fluorination of polymers - From fundamental research to industrial applications" Progress in Organic Coatings, vol. 61, issues 2-4, pp.192-204, February

[5] A. Tressaud, E. Durand, C. Labrugere, A.P. Kharitonov, L.N. Kharitonova "Modification of surface properties of carbon-based and polymeric materials through fluorination routes: From fundamental research to industrial applications" Journal of Fluorine Chemistry, vol. 128, pp378-391, January 2007

[6] Laurence W. McKeen "Fluorinated Coatings and Finishes Handbook" William Andrew Publishing, Inc. pp. 1-10

[7] Yue Jiang, Zhenlian An, Chenxia Liu, Feihu Zheng, Yewen Zhang "Influence of Oxyfluorination Time on Space Charge Behavior in Polyethylene" IEEE Transactions on Dielectrics and Electrical Insulation Vol. 17, No. 6, pp. 1814-1823, December 2010

[8] Z. An, Q. Yang, C. Xie, Y. Jiang, F. Zheng, Y. Zhang "Suppression of Charge Injection to Linear Low Density Polyethylene by Surface Fluorination Modification" International Symposium on Electrical Insulating Materials, pp.368-371, September 2008

[9] http://www.mouldlife.net/ekmps/shops/mouldlife/resources/Other/ly556-917-dy-070.pdf

[10] University of California Riverside "Introduction to Energy Dispersive Xray Spectrometry (EDS)" Central Facility for Advanced Microscopy and Microanalysis, pp. 1

[11] F.J. du Toit, R.D. Sanderson "Surface Fluorination of polypropylene:1. Characterisation of surface properties" Journal of Fluorine Chemistry, vol. 107, pp. 107-115, 1999

[12] A. Mohamad, G. Chen, Y. Zhang and Z. An "Surface Fluorinated Epoxy Resin for High Voltage DC Application" IEEE Transactions on Dielectrics and Electrical Insulation, October 2014

[13] A. Mohamad, G. Chen, Y. Zhang and Z. An "Influence of fluorination treatment on surface flashover of polymeric insulation" Conference on Electrical Insulation and Dielectric Phenomena (CEIDP), pp. 482-485, October 2013

[14] Josef Kindersberger, Christoph Lederle "Surface Charge Decay on Insulators in Air and Sulfurhexafluoride - Part II: Measurements" IEEE Transactions on Dielectrics and Electrical Insulation, vol. 15, issue 4, pp.949-957, August 2008

[15] P Molinie, M Goldman, J Gatellet "Surface potential decay on coronacharged epoxy samples due to polarization processes" Journal of Physics D: Applied Physics, vol. 28, no. 8, pp. 1601-1610, August 1995 